EDITOR'S

\title{
Adjunctive use of intravitreal dexamethasone in presumed bacterial endophthalmitis: a randomised trial
}

\author{
E Albrecht, ${ }^{1}$ J C Richards, ${ }^{2}$ T Pollock, ${ }^{3}$ C Cook, L Myers ${ }^{1}$
}

${ }^{1}$ University of Cape Town Groote Schuur Hospital, Cape Town, South Africa

${ }^{2}$ Royal Perth Hospital, Perth, Western Australia, Australia ${ }^{3}$ Sheikh Khalifa Medical City, Abu Dhabi, United Arab Emirates

\section{Correspondence to}

Dr Eric Albrecht, Department of Ophthalmology, University of Cape Town, Ward D4, Groote Schuur Hospital, 13 East Light Way, Kirstenhof 7945, Cape

Town, South Africa;

albrecht@telkomsa.net

Accepted 31 October 2010

Published Online First

2 February 2011

\section{ABSTRACT}

Aim To evaluate the use of intravitreal dexamethasone as adjunctive therapy in the treatment of presumed bacterial endophthalmitis.

Design Prospective, double masked, randomised placebo-controlled clinical trial.

Methods Patients with 'post cataract surgery', 'bleb-related' and 'other' endophthalmitis were grouped and randomised to receive intravitreal ceftazidime $(2.225 \mathrm{mg} / 0.1 \mathrm{ml})$, vancomycin $(1 \mathrm{mg} / 0.1 \mathrm{ml})$, and either dexamethasone $(0.4 \mathrm{mg} / 0.1)$ or placebo. All underwent vitreous and aqueous sampling for microbiological analysis. Injections were repeated after $48 \mathrm{~h}$ if necessary. The primary outcome measure was Snellen visual acuity on presentation, within the first 14 days post injection, and at 2-4 months.

Results 62 patients completed the protocol from 2001 to 2005. Thirty patients received intravitreal dexamethasone and 32 received intravitreal placebo. There was no statistically significant difference in the visual outcomes of either group with a mean 2.79 Snellen lines improvement of the intravitreal dexamethasone group versus 1.8 lines in the placebo group. Subgroup analysis suggested a clinical trend to better visual acuity in the post cataract steroid subgroup with mean 4.1 lines improvement versus 2.7 in the placebo group $(p=0.33)$. No adverse events attributable to the dexamethasone were reported.

Conclusions Intravitreal dexamethasone appears safe and may be of benefit in post cataract surgery bacterial endophthalmitis.

\section{INTRODUCTION}

Endophthalmitis is defined as inflammation of the contents or cavity of the eye and usually represents an infection of the vitreous. It has been theorised that any effective treatment should aim at treating both the infection and the inflammatory response. Intravitreal antibiotic injections are the mainstay of treatment. The role of any steroid either orally or intravitreally has been debated in the literature, and was first the subject of experiment in $1974 .{ }^{1}$ Steroid use remains controversial.

A survey of all post cataract endophthalmitis in the $\mathrm{UK}^{2}$ showed that of the 213 patients, only $17 \%$ received intravitreal steroids. This practice reflects the lack of clear guidelines from the literature.

In a 2002 'Clinical controversy' review, Elder and Morlet ${ }^{3}$ concluded that there was no clear evidence either for or against the adjunctive use of intravitreal steroids.

Shah et $a l^{4}$ in 2000 reported a retrospective nonrandomised comparative trial of 57 postoperative endophthalmitis cases comparing adjunctive intravitreal steroids with intravitreal antibiotics alone.
Patients who received steroids had a significantly reduced likelihood of obtaining a 3-line improvement.

Gan et $a l^{5}$ reported a prospective randomised placebo controlled clinical trial $(n=29)$ of postoperative endophthalmitis, comparing adjunctive intravitreal dexamethasone to placebo in addition to the intravitreal antibiotics with outcomes measured at 3 and 12 months. This small study was the first randomised one to demonstrate a trend towards a better visual acuity in the dexamethasone group, justifying further investigation.

The dose of intravitreal dexamethasone has become standardised since the report of Kwak et al ${ }^{6}$ in 1992 in which histology suggested increasing disorganisation of the Muller cells at doses above $440 \mu \mathrm{g}$.

\section{METHODS}

\section{Study design}

A prospective double-masked randomised clinical trial was undertaken comparing the adjunctive use of intravitreal dexamethasone versus placebo in addition to standard intravitreal antibiotics in presumed bacterial endophthalmitis.

\section{Participants}

All patients with presumed bacterial endophthalmitis presenting to Groote Schuur Hospital were considered for inclusion. They were divided into three aetiological groups by the admitting clinician: post cataract (PC), bleb-related endophthalmitis (GB) and other (O), which included post penetrating injuries, endogenous endophthalmitis and post pars plana vitrectomy. Post cataract endophthalmitis was selected as a priority subgroup, for comparison with similar groups in other trials.

\section{Exclusion criteria}

1. Suspected fungal/parasitic/viral/non-bacterial endophthalmitis.

2. Patients who underwent vitrectomy for the endophthalmitis were not recruited.

'Our very few patients who underwent vitrectomy for endophthalmitis (ideally those with visual acuity of hand movement (HM) according to the Endophthalmitis Vitrectomy Study) were not recruited because:

1. Understaffing of our vitreoretinal service during the period of the trial meant that we could not offer vitrectomy to most eligible cases (in general even patients with HM vision or worse would be treated only with intravitreal injections)

2. In those rare patients who were offered a vitrectomy we felt that the results at 3 months would not be comparable with the unvitrectomised subjects.

3. Numbers would have been so small that we could not derive meaningful statistical information from the group.

4. Vitrectomised patients would have been no more or less likely than unvitrectomised patients to be randomised to a particular group so that their exclusion would not confound our analysis. 


\section{Intervention}

On presentation, patients were admitted and counselled, informed consent was obtained and intravitreal antibiotics and dexamethasone/placebo ordered. The pharmacy randomised the patients within the three groups using standard computer generated randomisation tables, to receive either dexamethasone $0.4 \mathrm{mg} / 1 \mathrm{ml}$ or placebo $0.1 \mathrm{ml}$ balanced salt solution, with the standard vancomycin $1 \mathrm{mg} / 0.1 \mathrm{ml}$ and ceftazidime $2.225 \mathrm{mg} /$ $0.1 \mathrm{ml}$. Patients who were allergic to penicillin received amikacin $0.4 \mathrm{mg} / 1 \mathrm{ml}$ in place of ceftazidime. A double-blinding label (dexamethasone/placebo) masked the dexamethasone/placebo injection to both surgeon and patient.

Vitreous and aqueous samples were sent for microbiological analysis. A subconjunctival injection of vancomycin $(25 \mathrm{mg} /$ $0.5 \mathrm{ml}$ ), ceftazidime $(50 \mathrm{mg} / 0.5 \mathrm{ml})$ and betamethesone $(1.5 \mathrm{mg} /$ $0.5 \mathrm{ml}$ ) was also administered at the end of the procedure.

Post injection, patients received topical ofloxacin and topical dexamethasone. Patients were re-injected after $48-72 \mathrm{~h}$ if needed.

\section{Outcomes}

The primary outcome measure was the visual acuity using standard Snellen chart and visual acuity worse than $6 / 60$ was graded using no perception of light, perception of light, hand movements, and count fingers at $1 \mathrm{~m}$. The visual acuity was then grouped into the following categories for comparison:

Group 1: $6 / 6-6 / 18$

Group 2: $6 / 24-6 / 60$

Group 3: $<6 / 60$

The visual acuities at presentation and at 3 months, as well as the number of lines improvement on the Snellen chart, were recorded.

The secondary outcome measures were any adverse events and any side effects of the medication.

\section{Statistical analysis}

On completion of the study the pharmacy master records were unmasked, and the data collected from a standard form in the patient folders. Data were entered using a custom-designed template in Microsoft Excel and analysed using Stata Version 9.0. The analysis was on an intention-to-treat basis and was stratified according to the underlying cause of the endophthalmitis. Variables were described using means, medians and proportions, as appropriate. Bivariate comparisons were based on Student $t$ test (for means), Wilcoxon sum rank test (for medians) and $\chi^{2}$ or Fisher's exact test (for proportions). The main analysis focused on describing the difference in the visual acuity outcome in the two groups. All statistical tests were two sided at $\alpha=0.05$.

\section{RESULTS}

Sixty-two were patients were enrolled between January 2001 and December 2005. Figure 1 shows the study flow diagram.

Table 1 compares some of the characteristics of the two groups. There were no significant differences in any of the characteristics between the two groups.

Of the 62 patients, 30 received intravitreal steroid while 32 received intravitreal placebo. Figure 2 shows the proportion of each subgroup receiving steroid or placebo. The largest subgroup was the post cataract surgery group (PC), comprising 32 of the total 62 patients of whom 15 received intravitreal placebo and 17 received intravitreal steroid. There were 13 patients with bleb-related endophthalmitis of whom four patients received

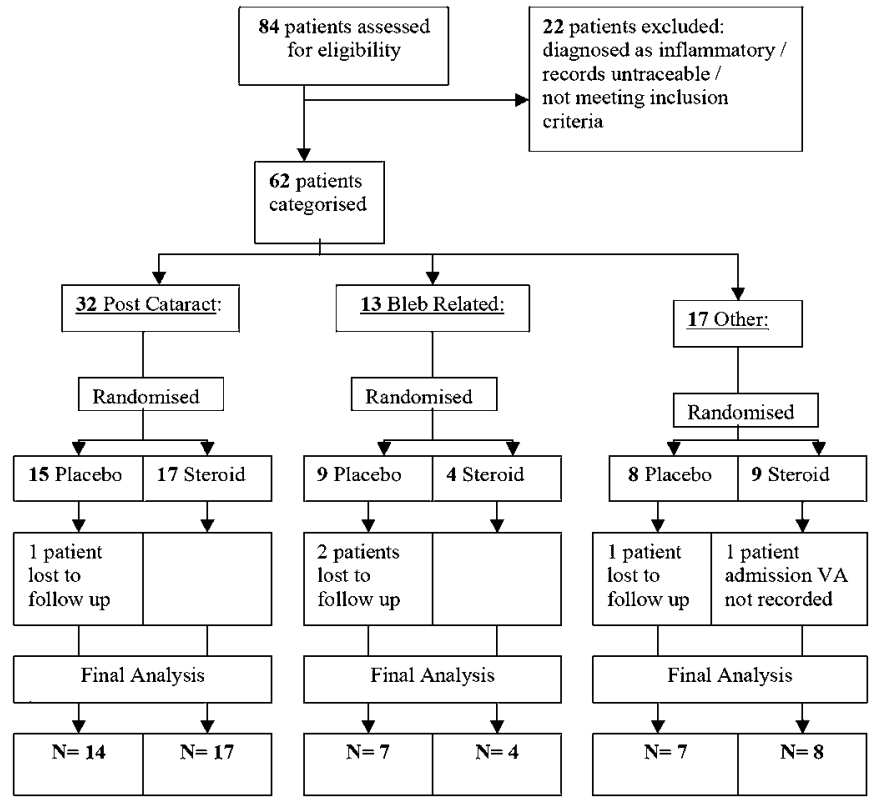

Figure 1 Study flow diagram.

intravitreal steroid while nine patients received intravitreal placebo. Seventeen patients were classified as Other: eight trauma-related with four receiving intravitreal steroids and four

Table 1 Baseline demographics

\begin{tabular}{|c|c|c|c|}
\hline & Steroid & Placebo & p Value \\
\hline Mean age (years) & $59(29-91)$ & $61(20-83)$ & $>0.5$ \\
\hline Gender (male) (n (\%)) & $11(36)$ & $18(56)$ & 0.137 \\
\hline \multicolumn{4}{|l|}{ Group (n (\%)) } \\
\hline Post cataract & $17(56)$ & $15(47)$ & 0.974 \\
\hline Post bleb & $4(13)$ & $9(28)$ & \\
\hline Other & $9(30)$ & $8(25)$ & \\
\hline \multicolumn{4}{|l|}{ Systemic comorbidity (n (\%)) } \\
\hline Yes & $12(40)$ & $15(47)$ & 0.585 \\
\hline No & $18(60)$ & $17(53)$ & \\
\hline \multicolumn{4}{|l|}{ Diabetes mellitus (n (\%)) } \\
\hline Yes & $6(20)$ & $11(34)$ & 0.260 \\
\hline No & $24(80)$ & $21(66)$ & \\
\hline \multicolumn{4}{|l|}{ Hypertension (n (\%)) } \\
\hline Yes & $4(13)$ & $6(19)$ & 0.733 \\
\hline No & $26(87)$ & $26(81)$ & \\
\hline \multicolumn{4}{|l|}{ HIV (n (\%)) } \\
\hline Yes & $1(3)$ & $1(3)$ & 0.999 \\
\hline No & $29(97)$ & $31(97)$ & \\
\hline \multicolumn{4}{|l|}{ Ocular comorbidity (n (\%)) } \\
\hline Yes & $10(33)$ & $15(46)$ & 0.311 \\
\hline No & $20(66)$ & $17(54)$ & \\
\hline \multicolumn{4}{|l|}{ Glaucoma (n (\%)) } \\
\hline Yes & $5(17)$ & $11(34)$ & 0.150 \\
\hline No & $25(83)$ & $21(66)$ & \\
\hline \multicolumn{4}{|l|}{ Diabetic retinopathy (n (\%)) } \\
\hline Yes & $5(17)$ & $2(6)$ & 0.249 \\
\hline No & $25(83)$ & $30(94)$ & \\
\hline \multicolumn{4}{|l|}{ Cataract (n (\%)) } \\
\hline Yes & $2(7)$ & $1(3 \%$ & 0.607 \\
\hline No & $28(93)$ & $31(97)$ & \\
\hline \multicolumn{4}{|l|}{ Age-related macular degeneration (n (\%)) } \\
\hline Yes & 0 & $2(6)$ & 0.492 \\
\hline No & $30(100)$ & $30(94)$ & \\
\hline \multicolumn{4}{|l|}{ Presenting visual acuity $(\mathrm{n} / \mathrm{n}(\%))$} \\
\hline Proportion with count fingers or less & 23/29 (79) & $28 / 32(87.5)$ & 0.496 \\
\hline
\end{tabular}




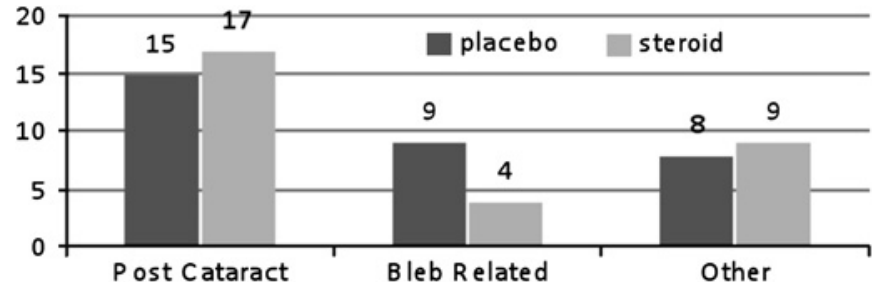

Figure 2 Subgroups of steroid and placebo.

receiving intravitreal placebo; three endogenous endophthalmitis of whom one received intravitreal steroid and two received intravitreal placebo; six endophthalmitis following pars plana vitrectomy of whom four received intravitreal steroids and two received intravitreal placebo.

The primary outcome was Snellen visual acuity on admission and at 3 months. The number of lines improvement on the Snellen visual acuity chart was compared. This ranged from -3 , that is lost 3 lines, to 9 , that is gained 9 lines, which included lines of no perception of light, perception of light, hand movements and count fingers, as well as the standard Snellen visual acuities. The mean improvement in the placebo group was 1.79 lines (range -3 to 9) compared with the steroid group, which showed 2.76 (range -3 to 9) lines of improvement (Student $t$ test, $\mathrm{p}=0.285$ ).

To compare visual outcomes between the two groups the Snellen visual acuities were also grouped into three categories: Group 1: Good visual outcome 6/6-6/18

Group 2: Visually impaired: $6 / 24-6 / 60$

Group 3: Severe visual impairment and blindness: less than $6 / 60$, that is count fingers to no perception of light.

Figure 3 shows the non-significant difference between visual outcomes at 3 months of the total steroid and the total placebo group with a $\mathrm{p}$ value of 0.757 (Fisher's exact test).

Analysis of the post cataract group, consisting of 17 steroid patients and 15 placebo patients, showed a similar lack of significant difference. Mean improvement in Snellen visual acuity was 2.7 lines ( -3 to 9 ) for the placebo group compared with 4.1 ( -3 to 9) for the steroid group (Student t test, $\mathrm{p}=0.330)$.

In the post cataract placebo group, 31\% (4/13) had a good visual outcome with a visual acuity of $6 / 18$ or better compared with the steroid group where $65 \%(11 / 17)$ had a visual acuity of $6 / 18$ or better. Figure 4 shows the 3 -month comparison in Snellen category between the placebo and steroid patients in the post cataract subgroup, which shows a $p$ value of 0.214 (Student $t$ test).

In the bleb-related endophthalmitis group, nine patients received intravitreal placebo, of whom two patients did not attend for follow-up and four received intravitreal steroids. The

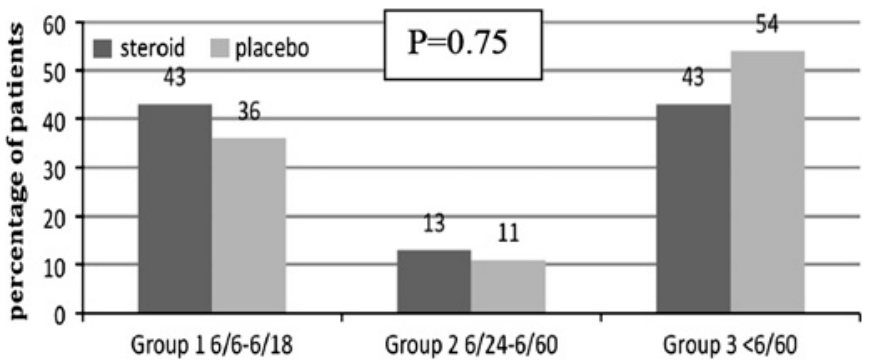

Figure 3 Three-month visual acuity comparison between placebo and steroid.

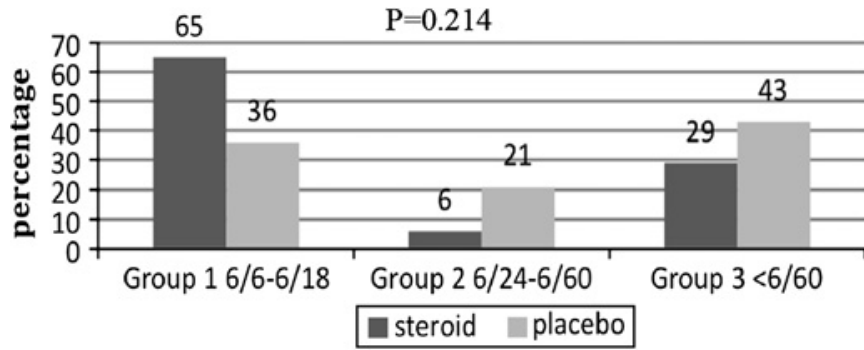

Figure 4 Post cataract 3-month visual acuity placebo versus steroid.

mean number of lines improvement in Snellen visual acuity for this placebo subgroup was 0.85 lines compared with 1.25 lines in the steroid subgroup (Student t test, $\mathrm{p}=0.95$ ).

Of those patients who were classified as "other":

- eight had trauma related endophthalmitis of whom four received steroid and four received placebo.

- three had endogenous endophthalmitis of whom one received steroid and two received placebo.

- six had post pars plana vitrectomy endophthalmitis of whom four received steroid and two received placebo.

The mean number of lines of improvement in Snellen acuity in the Placebo-Other subgroup was 0.714 compared with 0.625 lines in the Steroid-Other subgroup (Student t test, $\mathrm{p}=0.851$ ).

The vitreous/aqueous taps yielded a $52.5 \%$ positive culture rate. The most common organism cultured was Staphylococcus epidermidis, in $23 \%$ of all cases, followed by Staphylococcus aureus and Streptococcus species (including pneumoniae, mitis, oralis, constellatus, viridans and intermedius). Unfortunately five results were lost with the installation of a new hospital information system.

The only adverse events were three rhegmatogenous retinal detachments, all following complicated cataract surgery and all following use of intravitreal steroids. We could not attribute any adverse reactions directly to the intravitreal steroids.

We noted a delay in the presentation of the post cataract patients, of whom three presented with chronic endophthalmitis at 2 months, 5 months and 6 months, respectively. The mean delay in presentation was 20.25 days. If the three chronic cases were excluded, the mean delay was 8.6 days.

We combined the results of this study with the individual patient data presented on the same association by Gan et al. ${ }^{5}$ Sixty patients were included in this analysis ( 32 from this study, 28 from the Gan paper), with 29 and 31 subjects exposed to steroid and placebo, respectively. In the combined steroid group, $38 \%$ of patients experienced poor visual acuity outcome $(<6 / 18)$ compared with $68 \%$ of patients in the placebo group, resulting in a relative risk of $0.52(95 \% \mathrm{CI} 0.29$ to $0.93, p=0.021)$. In the same merged analysis, the benefit of steroid was also observed in the mean lines improvement, with steroid use associated with an average of 1.8 lines better improvement compared with placebo (t test $\mathrm{p}=0.08$ ).

\section{DISCUSSION \\ Limitations}

The most important limitation of the study was the small sample size, which could have been improved with a multicentre study. We had only $25 \%$ power to detect a statistically significant different between those randomised to steroid and placebo in the post cataract subgroup. Although the results from this study did not achieve statistical significance, when we combined these findings with those of Gan et al, ${ }^{5}$ whose results 
were highly consistent, the resulting measure of the benefit of steroid in preventing poor visual outcome is clear. Although caution must be exercised in merging the data from two studies with slightly different methods, management practices and determination of outcomes, this analysis points to the likely value of steroids in post cataract endophthalmitis.

Second, during data analysis it was not possible to describe precise details of all the patients excluded from the study, as recommended by the CONSORT (www.consort-statement.org/) group. A third limitation was the use of Snellen visual acuity charts, the only type of chart available in our clinics. Holladay described use of the logarithm of minimum angle of resolution (logMAR) acuity in order to compare geometric means of different groups. ${ }^{7}$ He pointed out that there is no logMAR or geometric equivalent for visual acuity of light perception or no light perception, as these do not represent a measurable angle, but merely the detection or absence of a light stimulus. These categories, therefore, need to be reported separately. In our study, this means that $22(35 \%)$ patients would be analysed separately, making logMAR analysis of the remaining visual acuities meaningless.

A difficulty with all trials on this subject is in confirming the diagnosis of bacterial endophthalmitis and differentiating it from inflammatory uveitis, particularly in the early postoperative period. The gold standard is an appropriate vitreous or aqueous tap culture result. Our $52.5 \%$ positive culture result is in keeping with the international standard. ${ }^{8}$ The baseline comparative demographics (table 1) reveal no significant difference between the two groups. Successful randomisation should ensure equal effect of this potential confounding variable on each group.

Although longer follow-up would have been preferable, the use of the visual acuity at 3 months as a final acuity was a compromise between improving the follow-up rate (traditionally very poor in our community) and allowing sufficient time for the vision to stabilise while waiting for late complications to develop. We acknowledge that the early anti-inflammatory effect of the steroids could have allowed quicker recovery but no difference in final vision if measured at either 6 or 12 months. However Gan et al $l^{5}$ measured their outcomes at 12 months and the benefit remained consistent. Conversely the placebo group may have been at higher risk for long-term complications such as tractional retinal detachments, which may have been missed at the 3-month mark.

\section{Conclusions}

The visual outcome in comparing the total steroid with the total placebo group showed no significant difference, which is in keeping with other studies. A comparison of the priority subgroup of the post cataract patients with those in other studies is however, important. Although small numbers meant that the p value was only 0.330 , a clinically significant beneficial trend was demonstrated, with the average number lines improvement being 4.1 in the steroid group compared with 2.7 in the non-steroid group (range -3 to 9 in both groups). In contrast, patients receiving intravitreal steroids in Shah et al's non-randomised trial had worse visual outcomes than those receiving no steroids. ${ }^{4}$ Our results were in keeping with those of Gan et al's ${ }^{5}$ randomised trial of 29 patients, which showed a trend to better visual acuity in the steroid group and combined analysis showed a statistically significant benefit.

The role of intravitreal steroids was inconclusive in the blebrelated endophthalmitis group and the Other-endophthalmitis group as the very low numbers in each group resulted in $p$ values of nearly 1 . The number of lines improvement/deterioration and the final visual categories achieved in each group appeared clinically similar.

No adverse reactions or side effects were directly attributed to the intravitreal steroids. The only adverse events were the three rhegmatogenous retinal detachments in patients who had complicated cataract surgery.

Our mean delay to presentation of 20.25 days was surprisingly long and even removing the three chronic cases still left a mean delay of 8.6 days. This was probably attributable to factors such as poor education and lack of access to tertiary hospitals.

\section{Recommendations}

Consideration should be given to using intravitreal dexamethasone in the treatment of postoperative endophthalmitis. It has been shown to be safe and probably beneficial in two very similar randomised studies.

The delayed presentation of our patients highlighted the need for patient education regarding the symptoms of endophthalmitis. We have adapted our standard post cataract surgery data sheets to include a tick box that both reminds the surgeon to highlight the dangers to the patient and to document that the patient has been informed. An improved referral system from our secondary level units performing high volume cataract surgery has also been implemented.

Acknowledgements We would like to acknowledge Drs JC Richards and T Pollock who designed and initiated the study, Professor C Cook for supervising the trial, Professor $\mathrm{L}$ Myers for the statistical analysis, and all the hardworking Registrars, Consultants and Nursing Staff of the Ophthalmology Division without whose assistance this would not have been possible.

Competing interests None to declare.

Patient consent Obtained.

Ethics approval Approval from the University Of Cape Town Research Ethics Committee, Faculty of Medicine, was formally granted on 9 February 2001, REC REF: 226/2000

Provenance and peer review Not commissioned; externally peer reviewed.

\section{REFERENCES}

1. Graham Ro, Peyman GA. Intravitreal injection of dexamethasone. Treatment of experimentally induced endophthalmitis. Arch Ophthalmol 1974;92:149-54.

2. Kamalarajah S, Silvestri G, Sharma N, et al. Surveillance of endophthalmitis following cataract surgery in the UK. Eye 2004;18:580-7.

3. Elder MJ, Morlet N. Clinical controversy. Clin and Exp Ophthalmol 2002:30:394-8.

4. Shah GK, Stein JD, Sharma S, et al. Visual outcomes following the use of intravitreal steroids in the treatment of postoperative endophthalmitis. Ophthalmology 2000; 107:486-9.

5. Gan IM, Ugahary LC, van Dissel JT, et al. Intravitreal dexamethasone as adjuvant in the treatment of postoperative endophthalmitis: a prospective randomized trial. Graefes Arch Clin Exp Ophthalmol 2005;243:1200-5.

6. Kwak HW, D'Amico DJ. Evaluation of the retinal toxicity and pharmacokinetics of dexamethasone after intravitreal injection. Arch Ophthalmol 1992;110:259-66.

7. Holladay JT. Visual acuity measurements. J Cataract Refract Surg 2004;30:287-90

8. Endophthalmitis Vitrectomy Study Group. Results of the Endophthalmitis Vitrectomy Study: a randomized trial of immediate vitrectomy and of intravenous antibiotics for the treatment of postoperative bacterial endophthalmitis. Arch Ophthalmol 1995;113:1479-96 